\title{
Non-Basal Dislocation Nucleation Site of Solid Solution Magnesium Alloy
}

\author{
Hidetoshi Somekawa ${ }^{1,4, * 1}$, Dudekula Althaf Basha ${ }^{1, * 2}$, Alok Singh ${ }^{1}$, Tomohito Tsuru ${ }^{2,4}$ and \\ Masatake Yamaguchi ${ }^{3,4}$ \\ ${ }^{1}$ Research Center for Structural Materials, National Institute for Materials Science, Tsukuba 305-0047, Japan \\ ${ }^{2}$ Nuclear Science and Engineering Center, Japan Atomic Energy Agency, Tokai-mura, Ibaraki 319-1195, Japan \\ ${ }^{3}$ Center for Computational Science and e-Systems, Japan Atomic Energy Agency, Tokai-mura, Ibaraki 319-1195, Japan \\ ${ }^{4}$ Elements Strategy Initiative for Structural Materials, Kyoto University, Kyoto 606-850, Japan
}

\begin{abstract}
The effect of grain boundary segregation on plastic deformation was investigated using the Mg-Y solid solution binary alloy. Deformed microstructural observations revealed many traces of prismatic $\langle\boldsymbol{a}\rangle$ dislocations as well as basal dislocations. These dislocations were nucleated at grain boundaries with segregation of yttrium element. By comparison of material factors of binary alloy, the alloying elements having low critical resolved shear stress (CRSS) of non-basal plane and large grain boundary (twin boundary) segregation energy led to activation of non-basal dislocations in the vicinity of grain boundaries. The $\mathrm{Mg}-\mathrm{Ca}$ alloy had similar material factors and showed the same deformed microstructures as those of the alloys containing rare-earth element, which indicate that calcium element is an alternative alloying element. [doi:10.2320/matertrans.MT-M2020040]
\end{abstract}

(Received January 30, 2020; Accepted March 12, 2020; Published April 17, 2020)

Keywords: magnesium, metals and alloys, grain boundaries, segregation, dislocation slip, alloying

\section{Introduction}

Magnesium $(\mathrm{Mg})$ and its alloys have been paid great attention in the structural parts and fields, due to having the lowest density among conventional metallic materials. Alloying of metallic materials has been the most well-used method to improve the properties. With no exception to $\mathrm{Mg}$, this common approach has been recognized as an important way. In particular, alloying with rare-earth (RE) elements is reported to cause unique properties and characteristics. ${ }^{1,2)}$ For instance, the Mg-RE alloys show not only high strength, enhanced creep resistance and corrosion resistance, but also they have the weaken basal texture, low stacking fault energies and low difference in critical resolved shear stress (CRSS) of basal vs. non-basal planes. Numerical studies have pointed out that these unique behaviors are closely related to the electric interactions in $d$-orbits (outermost electron shell) of RE elements and $p$-orbits of Mg. ${ }^{3)}$

As well as showing these properties and characteristics of the $\mathrm{Mg}-\mathrm{RE}$ alloys, $\mathrm{RE}$ element is likely to show grain boundary segregation behavior. ${ }^{4-8)}$ Grain boundary segregation in the metallic materials is an important microstructural factor which affects deformation behavior and mechanical properties. As for the $\mathrm{Mg}$ alloys adding non-RE element, grain boundary segregation of manganese $(\mathrm{Mn})$ atom leads to enhance room-temperature grain boundary sliding., $\left.{ }^{9}, 10\right)$ Regarding $\mathrm{Mg}-\mathrm{RE}$ alloys, grain boundaries at which $\mathrm{RE}$ atoms segregate play a role in prevention of grain boundary sliding. ${ }^{4,8)}$ In addition, such segregated boundaries become the crack propagation routes in the case of using crackinduced specimens. ${ }^{11)}$ On the contrary, irrespective of gain boundary segregation behavior, grain boundaries themselves are well-recognized as to become sink and source sites for

\footnotetext{
${ }^{* 1}$ Corresponding author, E-mail: SOMEKAWA.Hidetoshi@nims.go.jp

${ }^{*}$ Present address: Metallurgy Engineering and Materials Science, Indian Institute of Technology Indore, India
}

dislocation. ${ }^{12-14)}$ In particular, grain boundaries of $\mathrm{Mg}$ alloy are pointed out to affect deformation mechanism, i.e., operation of grain boundary compatibility; ${ }^{15)}$ nevertheless, there is still no clarity and reports about the effect of grain boundary segregation on plastic deformation, focusing on dislocation slips. This is probably due to that grain boundary structures are readily changed with plastic deformation progress. In this study, we have observed deformed microstructures at the beginning stage of plastic deformation, i.e., a quite low applied strain to specimen, using the $\mathrm{Mg}-\mathrm{Y}$ solid solution alloy. Furthermore, since the use of RE element has a possibility of increasing the cost and price, we have considered influential material factors of the binary alloys to seek for the alternative alloying elements for obtaining the same deformation behavior.

\section{Experimental Procedures}

A solid solution $\mathrm{Mg}-0.3$ at\% $\mathrm{Y}$ binary alloy produced by hot extrusion was used in this study. The extrusion temperature and ratio were $598 \mathrm{~K}$ and $25: 1$, respectively. The extruded alloy was annealed to annihilate strains/ dislocations at $473 \mathrm{~K}$ for 24 hours. While the microstructure is not shown here, the annealed alloy consisted of recrystallized microstructure having grain sizes of $\sim 5 \mu \mathrm{m}$. Tensile test was performed on this annealed alloy to prepare a deformed specimen at room-temperature. The initial strain rate was $1 \times 10^{-3} / \mathrm{s}$ and the applied strain was $0.015(=1.5 \%)$. Tensile direction was parallel to the extrusion direction. The microstructures of the deformed specimen were observed by high-resolution electron microscopy (HREM) and transmission electron microscopy (TEM). The specimen for TEM and HREM observations were prepared by ion milling.

\section{Results and Discussion}

Deformed microstructures of the Mg-0.3Y alloy are shown in Fig. 1. Figure 1(a) is the scanning TEM low-angle annular 

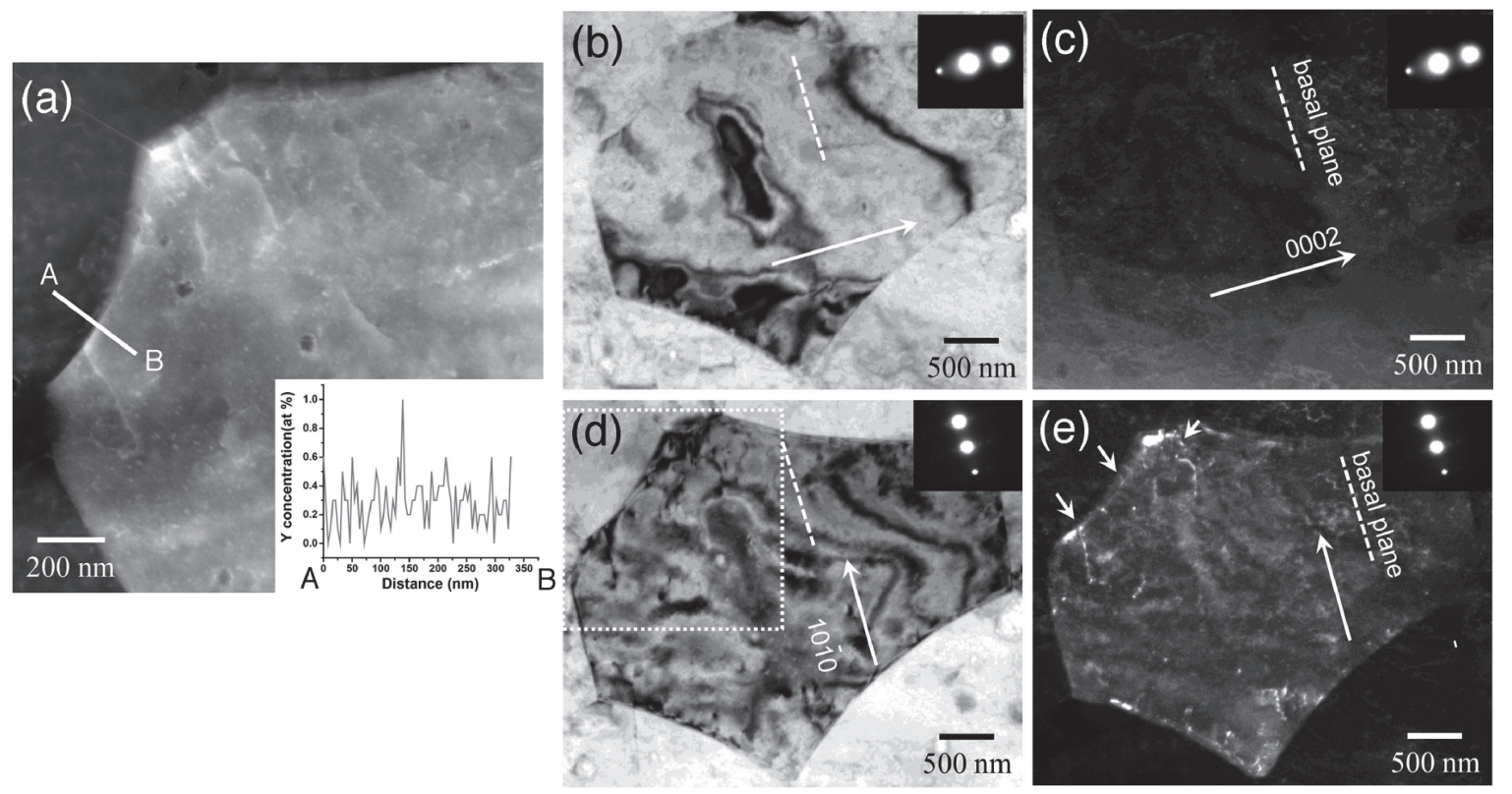

Fig. 1 Deformed microstructures in the vicinity of grain boundaries in the $\mathrm{Mg}-0.3 \mathrm{Y}$ alloy; (a) low-angle annular dark-field image including nano-EDX line profiled analysis, (b), (c) the weak beam bright- and dark-field images taken under two-beam diffraction conditions $(\boldsymbol{g}$ - $3 \boldsymbol{g})$ using different diffraction vectors, $\boldsymbol{g}=(0002)$ and (d), (e) $\boldsymbol{g}=(10 \overline{10})$. White arrows in Fig. (e) indicate $\langle\boldsymbol{a}\rangle$ type prismatic dislocations.

dark-field (LAADF) image, showing Z- and diffraction contrasts. A nano-energy dispersive X-ray spectroscopy (EDX) line profile of marked A-B is inset in this image. This observed area (as provided in Fig. 1(a)) corresponds to the white-dashed enclosed region in Fig. 1(d). Although the chemical composition of this alloy is 0.3 at\%, the EDX analysis obtained at grain boundary has a high peak of more than 1.0 at\%. In the Z-contrast image, grain boundaries are also found to be bright in contrast. These results display that yttrium $(\mathrm{Y})$ atom segregates at grain boundaries, which are well-observed in the thermomechanical processed Mg alloys containing RE elements. ${ }^{4-8)}$ Figures 1(b) to 1(e) are the TEM images in the vicinity of grain boundaries near zone axis of [1120]. Figures 1(b) and 1(d) are the respective two-beam bright-field images, and Figs. 1(c) and 1(e) are their corresponding $(\boldsymbol{g}-\mathbf{3} \boldsymbol{g})$ weak-beam dark-field images taken using diffraction vectors, $\boldsymbol{g}=(0002)$ and $(10 \overline{1} 0)$. Some of dislocation slips marked by small white arrows are clearly confirmed in the vicinity of grain boundaries in Fig. 1(e); in contrast, such features are unlikely to be observed in Fig. 1(c). In accordance with $\boldsymbol{g} \cdot \boldsymbol{b}$ criterion, these dislocations are the basal and prismatic $\langle\boldsymbol{a}\rangle$ type dislocations. Hence, grain boundary is assumed to be the origin for these dislocations, i.e., the basal and non-basal dislocations. Sandlobes et al. ${ }^{16)}$ have pointed out that non-basal dislocations are nucleated at the stacking fault in the $\mathrm{Mg}-\mathrm{Y}$ alloys, because the addition of RE elements decreases the stacking fault energy. At the same time, Koike et al. ${ }^{15)}$ have reported that grain boundary compatibility plays a role in acceleration of nonbasal dislocation slips in conventional/commercial Mg alloys (without RE element alloying). This tendency of non-basal dislocation slip activities is well-observed in deformed $\mathrm{Mg}$ alloys, ${ }^{17-19)}$ and is consistent with the present deformed microstructural observations. Beside this point, it is noted that $\mathrm{Y}$ atom segregates at grain boundaries in Fig. 1(a), which seems to affect dislocation slip. From the atomistic viewpoint, grain boundaries that are segregated with solute atom are incoherent and have atomistic ordered steps. ${ }^{20,21)} \mathrm{In}$ order to maintain plastic deformation, such heterogeneous grain boundaries have a possibility to create large stress field even in the beginning deformation stage; as a result, readily nucleating the dislocations.

Hereafter, focusing on material factors, the alloying impact is considered to make a search of alternative alloying element. According to thermodynamics, segregation behavior occurs to stabilize and to reduce the total energy. In general, solute atom is likely to segregate into the bulk to form precipitate, at the external surfaces to form surface layers, and at the internal interfaces such as grain/twin boundaries. In this study, since the chemical composition of 0.3 at $\%$ in $Y$ element is much lower than that of maximum solubility, ${ }^{22)}$ precipitation is assumed to be ruled out. As for the second point, surface layer segregation is not considerable factor, due to non-formation of new surfaces associated with fracture and/or crack propagation. In contrast, grain boundary segregation is clearly confirmed in this alloy; thus, segregation energy for grain/twin boundary is used as an influential material factor, in this discussion. A part from segregation behavior, CRSS is closely related to the dislocation slip activities. It is commonly recognized that alloying element which reduces the difference in CRSS between the basal and the non-basal planes brings about highly enhancement of non-basal dislocation slips. ${ }^{23-26)}$ Based on these observations, the relationship between CRSS of non-basal plane and twin boundary segregation energy in several binary alloys is shown in Fig. 2. These values are cited from our previous studies obtained from first-principles calculations. ${ }^{27,28)}$ Although the actual bulked samples consist 


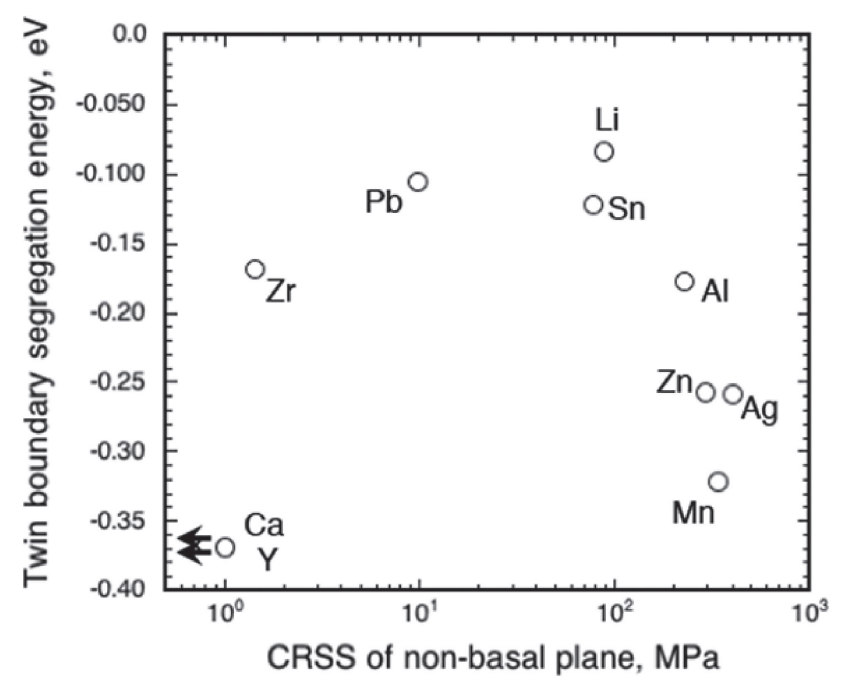

Fig. 2 The relationship between CRSS of non-basal plane and twin boundary segregation energy of binary alloys. These values are cited from our previous numerical studies via first-principles calculations. ${ }^{27,28)}$ Note that CRSS of $\mathrm{Y}$ and $\mathrm{Ca}$ elements are lower than $1 \mathrm{MPa}$.

of various types of grain boundaries, all or each types of grain boundary energies are unable to be obtained from experimental and numerical studies. Thus, in this study, twin boundary segregation energy is used, as a substitute for grain boundary segregation energy. This figure shows that $\mathrm{Y}$ element has the characteristic of low CRSS of non-basal plane and large segregation energy for twin boundary. It is obvious that calcium (Ca) element behaves similar to the $\mathrm{Y}$ element, which suggests that $\mathrm{Ca}$ atom segregated grain boundaries become the nucleation sites for non-basal dislocations. Deformed microstructural observations have reported that the $\mathrm{Mg}-\mathrm{Ca}$ solid solution alloy has the traces of non-basal dislocation slips in the matrix ${ }^{18,29)}$ and in the vicinity of grain boundaries (as provided in Appendix A). These results indicate that $\mathrm{Ca}$ is the impressive alternative alloying element.

Returning to the other alloying elements, zirconium $(\mathrm{Zr})$ element exhibits low CRSS of non-basal plane but low twin boundary segregation energy, as shown in Fig. 2. Alloying of this element has a possibility to activate non-basal dislocation slips, particularly, in the grain interior. However, it has been reported that $\mathrm{Zr}$ element forms precipitation in the wrought processed $\mathrm{Mg}-\mathrm{Zr}$ binary alloys. ${ }^{30)}$ That is due to low segregation energy and/or quite low solubility at wrought processed temperature ranges. ${ }^{22}$ ) The elements of Mn, silver (Ag) and zinc $(\mathrm{Zn})$ have the opposite trend, i.e., large segregation energy for twin boundary and high CRSS of nonbasal plane. Several studies have pointed out that the $\mathrm{Mg}-\mathrm{Mn}$ binary alloys show grain boundary segregation, and the other two elements ( $\mathrm{Ag}$ and $\mathrm{Zn}$ ) are also likely to segregate even at twin boundaries in each binary alloy. ${ }^{31)}$ The activation of non-basal dislocations are unlikely to be observed in these binary alloys, to the best of our knowledge; nevertheless, it is interesting to note that grain boundary segregation of $\mathrm{Mn}$ element plays a great role in enhancing grain boundary sliding, instead of being the nucleation site of non-basal dislocations.

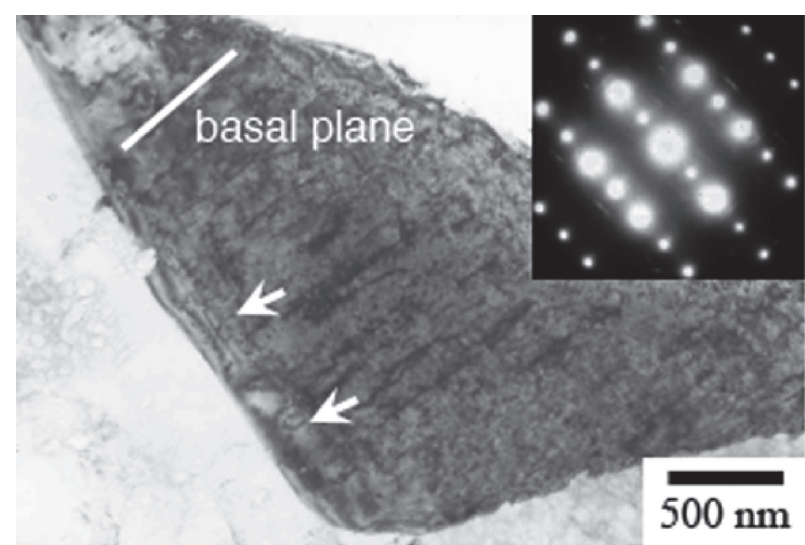

Fig. A1 Deformed microstructures of the meso-grained $\mathrm{Mg}-0.05 \mathrm{Ca}$ solid solution alloy. White arrows indicate the trace of non-basal dislocations.

\section{Summary}

Deformed microstructural observations showed the activation of basal and non-basal dislocations in the $\mathrm{Mg}-\mathrm{Y}$ solid solution alloy. Grain boundaries, at which Y atom segregate, had several traces of non-basal dislocation slips, i.e., prismatic $\langle\boldsymbol{a}\rangle$ dislocations. This indicated that such grain boundaries consisting of atomistic-ordered steps became the nucleation site for non-basal dislocations. Both CRSS of non-basal plane and grain boundary (or twin boundary) segregation energy were the effective judgement material factors to obtain the same grain boundary plasticity, i.e., nucleation of non-basal dislocations in the vicinity of grain boundaries.

\section{Appendix A}

Deformed microstructural observation via TEM was performed using the $\mathrm{Mg}-0.05$ at\% Ca solid solution alloy. This alloy was produced by extrusion and subsequent annealing to obtain the meso-grained structures of more than $50 \mu \mathrm{m}$ in size. Detailed procedure has been reported in our previous study. ${ }^{27)}$ Figure A1 is the bright field image taken by TEM including diffraction pattern. A high density of strain is confirmed in the vicinity of grain boundaries, since compressive strain of $0.20(=20 \%)$ was applied on the annealed $\mathrm{Mg}-\mathrm{Ca}$ alloy to prepare deformed specimen. Some of dislocation features as marked by white arrows are not parallel to the basal plane at grain boundaries; thus, they are found to be non-basal dislocations, which is the same as that of the present result of $\mathrm{Mg}-\mathrm{Y}$ alloy.

\section{Acknowledgements}

The authors are thanks to Ms. R. Komatsu and Ms. Y. Kobayashi (National Institute for Materials Science) for their technical help and acknowledge the support from the large-scale parallel computer system with SGI ICE X at Japan Atomic Energy Agency. This work was supported by the JSPS Grant-in-Aid (C) for Scientific Research in No. 16K06783 and 19K05068, and Scientific Research on Innovative Areas "MFS Materials Science" in No. $18 \mathrm{H} 05477$, and was partially supported by Element Strategy Initiative of MEXT in JPMXP0112101000. 


\section{REFERENCES}

1) D. Griffiths: Mater. Sci. Technol. 31 (2015) 10-24.

2) I. Basu and T. Al-Samman: Acta Mater. 96 (2015) 111-132.

3) T. Tsuru and D.C. Chrzan: Sci. Rep. 5 (2015) 8793.

4) H. Watanabe, A. Owashi, T. Uesugi, Y. Takigawa and K. Higashi: Philos. Mag. 92 (2012) 787-803.

5) J.D. Robson, S.J. Haigh, B. Davis and D. Griffiths: Metall. Mater. Trans. A 47 (2016) 523-530.

6) J.P. Hadorn, K. Hantzsche, S.B. Yi, J. Bohlen, D. Letzing, J.A. Wollmershauser and S.R. Agnew: Metall. Mater. Trans. A 43 (2012) 1347-1362.

7) M. Bugnet, A. Kula, M. Niewczas and G.A. Botton: Acta Mater. 79 (2014) 66-73.

8) H. Somekawa, A. Singh, T. Mukai and T. Inoue: Philos. Mag. 96 (2016) 2671-2685.

9) H. Somekawa: Mater. Trans. 61 (2020) 1-13.

10) H. Somekawa, H. Watanabe and T. Mukai: Philos. Mag. 94 (2014) 1345-1360.

11) H. Somekawa, T. Inoue and K. Tsuzaki: Philos. Mag. 95 (2015) 2513 2526.

12) M. Yoshiya and H. Yoshizu: Mater. Trans. 51 (2010) 51-57.

13) T. Tsuru, Y. Aoyagi and T. Shimokawa: Mater. Trans. 57 (2016) 14761481.

14) K. Kinoshita, T. Shimokawa and T. Kinari: Mater. Trans. 53 (2012) $147-155$.

15) J. Koike, T. Kobayashi, T. Mukai, H. Watanabe, M. Suzuki, K. Maruyama and K. Higashi: Acta Mater. 51 (2003) 2055-2065.

16) S. Sandlobes, S. Zaefferer, I. Schestakow, S. Yi and R. GonzalezMartinez: Acta Mater. 59 (2011) 429-439.
17) Y. Chino, M. Kado and M. Mabuchi: Acta Mater. 56 (2008) 387394.

18) K. Zhang, H. Wen, M.A. Kumar, F. Chen, L. Zhang, I.J. Beyerlein, J.M. Schoenung, S. Mahajan and E.J. Lavernia: Acta Mater. 120 (2016) 75-85.

19) S. Sandlobes, M. Friak, J. Neugebauer and D. Raabe: Mater. Sci. Eng. A 576 (2013) 61-68.

20) I.J. Beyerlein, M.J. Demkowicz, A. Misra and B.P. Uberuaga: Prog. Mater. Sci. 74 (2015) 125-210.

21) G. Duscher, M.F. Chisholm, U. Alber and M. Ruhle: Nat. Mater. 3 (2004) 621-626.

22) T.B. Massalski: Binary Alloy Phase Diagrams, 2nd edition, (ASM International, Materials Park, OH, 1990).

23) H. Yoshinaga and R. Horiuchi: Mater. Trans. JIM 4 (1963) 134-141.

24) K. Hayashi, M. Tsushida, H. Kitahara and S. Ando: Mater. Trans. 60 (2019) 1598-1600.

25) T. Tsuru, Y. Udagawa, M. Yamaguchi, M. Itakura, H. Kaburaki and Y. Kaji: J. Phys. Condens. Matter 25 (2013) 022202.

26) K. Hayashi, M. Tsushida, H. Kitahara and S. Ando: Mater. Trans. 60 (2019) 1598-1600.

27) H. Somekawa, M. Yamaguchi, Y. Osawa, A. Singh, M. Itakura, T. Tsuru and T. Mukai: Philos. Mag. 95 (2015) 869-885.

28) T. Tsuru, H. Somekawa and D.C. Chrzan: Acta Mater. 151 (2018) 7886.

29) H.C. Pan, H. Fu, B. Song, Y.P. Ren, C.Y. Zhao and G.W. Qin: Philos Mag. Lett. 96 (2016) 249-255.

30) H. Somekawa, D.A. Basha and A. Singh: Mater. Sci. Eng. A 766 (2019) 138384.

31) H. Somekawa, D.A. Basha, A. Singh, T. Tsuru and H. Watanabe: submitted. 